TITLE:

\title{
Significance of portal venous VEGF during liver regeneration after hepatectomy.
}

\section{$\operatorname{AUTHOR}(\mathrm{S}):$}

Yamamoto, Chiduru; Yagi, Shintaro; Hori, Tomohide; lida, Taku; Taniguchi, Kentaro; Isaji, Shuji; Uemoto, Shinji

\section{CITATION:}

Yamamoto, Chiduru ... [et al]. Significance of portal venous VEGF during liver regeneration after hepatectomy.. The Journal of surgical research 2010, 159(2): e37-e43

\section{ISSUE DATE:}

2010-04

URL:

http://hdl.handle.net/2433/113888

\section{RIGHT:}

(C) 2010 Elsevier B.V.; This is not the published version. Please cite only the published version.; この論文は出版社版でありません。引用の際に は出版社版をご確認ご利用ください。 


\section{Title page}

\section{Article title}

\section{Significance of Portal Venous VEGF During Liver Regeneration After Hepatectomy}

\section{Author's name}

Chiduru Yamamoto ${ }^{1)}$, M.Sci.

Shintaro Yagi ${ }^{2 \dagger}$, M.D., Ph.D

Tomohide Hori²), M.D., Ph.D

Taku Iida ${ }^{2)}$, M.D., Ph.D

Kentaro Taniguchi ${ }^{1)}$, M.D., Ph.D

Shuji Isaji ${ }^{1)}$, M.D., Ph.D

Shinji Uemoto²), M.D., Ph.D

\section{Affiliations:}

1) Department of Hepatobiliary and Pancreatic Surgery, Mie University Graduate school of medicine, 2-174 Edobashi Tsu City, Mie prefecture, 514-8507, Japan

2) Department of Hepatobiliary, Pancreas and Transplant Surgery, Kyoto University Graduate school of medicine, 54 Kawara-cho, Shogoin, Sakyo-ku, Kyoto city, Kyoto prefecture, 606-8507, Japan

Footnote: $\uparrow$ To whom correspondence and reprint requests should be addressed: Department of Hepatobiliary, Pancreas and Transplant Surgery, Kyoto University, 54 Kawara-cho, Shogoin, Sakyo-ku, Kyoto city, Kyoto prefecture, Japan 606-8507, Phone: +81-75-751-4323, Fax: +81-75-751-4348, E-mail: shintaro@kuhp.kyoto-u.ac.jp

Running title: Significance of portal venous VEGF in liver regeneration Subject category: gastrointestinal

The address for manuscript correspondence: Shintaro Yagi M.D., Ph.D. Department of 
Hepatobiliary, Pancreas and Transplant Surgery, Kyoto University, 54 Kawara-cho, Shogoin, Sakyo-ku, Kyoto city, Kyoto prefecture, Japan 606-8507, Phone: +81-75-751-4323, Fax: +81-75-751-4348, E-mail: shintaro@kuhp.kyoto-u.ac.jp 


\section{ABSTRACT}

Background. Although some studies have hypothesized portal venous blood is important for liver regeneration, no studies have established organs around the portal vein secrete liver regenerating factors into the portal vein during liver regeneration. The aim of this study was to elucidate up-regulation of vascular endothelial growth factor (VEGF) in the portal vein, and expressions of hepatic regenerating factors in organs around the portal vein during liver regeneration after partial hepatectomy (PHx).

Materials and Methods. VEGF protein in systemic and portal venous blood, and besides, expression of VEGF, hypoxia-inducible factor-1alfa (HIF-1 $\alpha$ ), hepatocyte growth factor (HGF) and HGF activator (HGFA) mRNA were evaluated in the regenerating liver, spleen and intestine following $70 \% \mathrm{PHx}$ in rats.

Results. The portal VEGF protein level was significantly higher than the systemic level post-PHx (portal /systemic at 72, 120 and $168 \mathrm{~h}$ post-PHx: 17.2/13.0, 20.2/12.8, and 24.0/14.7 pg/ml; $P=.003, P=.022$ and $P=.032$, respectively). VEGF mRNA expressions were significantly higher in the liver $(P=.000027$ : $168 h)$, spleen $(P=.000059: 72 h)$ and intestine ( $P=0.01$ : 24-72h) post-PHx compared to the pre-PHx levels. HIF-1 $\alpha$, HGF, HGFA mRNA expressions in the liver, intestine and spleen were also significantly higher post-PHx compared to the pre-PHx levels.

Conclusions. Portal VEGF was significantly higher than systemic VEGF, and besides expressions of VEGF, HIF-1 $\alpha$, HGF, and HGFA mRNA in the liver, spleen and intestine were up-regulated during liver regeneration. These results suggest that hepatic regenerating factors derived from the spleen or intestine may contribute liver regeneration.

Keywords: liver regeneration; portal vein; VEGF; HIF-1 $\alpha$; HGF; HGFA; spleen; intestine; 
partial hepatectomy 


\section{INTRODUCTION}

After extensive hepatectomy or segmental liver transplantation with a small-for-size graft, liver regeneration is essential for patient survival. Postoperative liver failure occurs because the small remnant liver volume cannot sustain the postoperative metabolic and synthetic demands, and remnant liver dysfunction can eventually lead to liver failure.

Liver regeneration is a highly complex and organized process that has been shown to involve the actions of a number of cytokines and growth factors, such as hepatocyte growth factor (HGF) (1), transforming growth factor- $\alpha(2)$, epidermal growth factor (3), hepatocyte growth factor activator (HGFA) (4) and vascular endothelial growth factor (VEGF) (5). Although some previous studies have hypothesized that liver regenerating factors secreted into the portal venous blood are important for liver regeneration (6-8), no studies have established that organs around the portal vein, such as the intestine, or spleen, actually secrete liver regenerating factors into the portal venous blood during liver regeneration. We have reported that the portal venous blood VEGF level was higher than the systemic venous blood VEGF level after living-donor liver transplantation (LDLT) (9) and hypothesized that portal venous VEGF secreted by the spleen or intestine plays an important role in graft regeneration, in addition to hepatocyte-derived VEGF. In the present study, we aimed to elucidate up-regulation of portal venous VEGF, and of hepatic regenerating factors in organs around the portal vein during liver regeneration after partial 
hepatectomy (PHx) in rats.

\section{Materials and Methods}

\section{Animals}

Male Wistar rats, weighing 250-300 g (10-13 the age of the week), were used. The animals were housed in a temperature- and humidity-controlled environment with a 12 hours light /12 hours dark cycle and allowed food (standard laboratory chow) and water ad libitum. All experiments were conducted in accordance with with the NIH guidelines, Principles of Laboratory Animal Care.

\section{Operative Procedure}

Under diethyl ether anesthesia, 70 \% PHx involving a median and left lateral lobectomy was performed according to the procedure of Higgins and Anderson (10). Animals were humanely sacrificed before and at 24, 72, 120 and 168 hours (h) after PHx, with twenty-five animals at each time point. For the assessment of VEGF, hypoxia-inducible factor- $1 \alpha$ (HIF-1 $\alpha)$, HGF and HGFA, the liver right inferior lobe, spleen and intestine (jejunum which is 3 centimeter apart from duodenum) were carefully excised and processed for different experimental procedures.

\section{Measurement of the Liver Wet Weight}

We calculated the ratio of liver regeneration at 24, 72, 120 and $168 \mathrm{~h}$ after PHx according to 
the following equations.

Ratio of liver regeneration (\%) = regenerating remnant liver (g) / estimated whole liver (g)

Estimated whole liver $(\mathrm{g})$ = resected liver $\mathrm{x}$ 100/70

\section{Analysis of VEGF, HIF-1 $\alpha$, HGF and HGFA Messenger Ribonucleic Acid (mRNA)}

\section{Expression by Reverse Transcription-Polymerase Chain Reaction (RT-PCR)}

The liver, spleen and small intestine were flash-frozen in liquid nitrogen immediately after harvesting and then stored at $-80^{\circ} \mathrm{C}$ until analysis. Total RNA was extracted from the tissues using an RNeasy Mini Kit (QIAGEN, Tokyo, Japan) according to the manufacturer's instructions. An aliquot of $5 \mu \mathrm{g}$ of the total RNA was then reverse-transcribed to complementary deoxyribonucleic acid (cDNA) using a SuperScript First-Strand Synthesis System (Invitrogen Japan KK, Tokyo, Japan). Next, the cDNA was amplified using specific primers for rat VEGF, rat HIF-1 $\alpha$, rat HGF or rat HGFA (Table 1). Reverse transcription and PCR amplification of a housekeeping gene, glyceraldehyde 3-phosphate dehydrogenase (GAPDH), were also performed to verify equal loading of the RNA and cDNA in the reverse transcription and PCR, respectively. The PCR was performed for 28-30 cycles under the following conditions: denaturation for 30 seconds at $94^{\circ} \mathrm{C}$, annealing for 30 seconds at $60^{\circ} \mathrm{C}$ and extension for 1 minute at $72^{\circ} \mathrm{C}$ in a program temperature control system. All the RT-PCR reaction products were electrophoresed in a $2 \%$ agarose gel and stained with ethidium bromide. 


\section{Blood Sample Collection and VEGF Assay}

Portal and systemic venous blood samples were obtained from the portal vein and inferior vena cava by direct puncture with a tuberculin needle in order to assess the VEGF levels before and at 24, 72, 120 and $168 \mathrm{~h}$ after PHx. Following collection of the samples into plasma separator tubes and centrifugation for 10 minutes at $3000 \mathrm{~g}$, the plasma samples were stored at $-80^{\circ} \mathrm{C}$ until analysis. The VEGF concentrations were measured using VEGF enzyme-linked immunosorbent assay (ELISA) kits (R\&D Systems, Minneapolis, MN) according to the manufacturer's instructions.

\section{Statistical Analysis}

The results were expressed as the means \pm standard deviation (SD). Differences among unpaired continuous or discontinuous data between two groups were analyzed using Student's t-test. All calculations were performed using StatView-J 5.0 statistical software (SAS Institute, Cary, NC).

\section{RESULTS}

\section{Liver Regeneration}

Figure. 1 shows the serial changes in the liver regenerative ratio during the $168 \mathrm{~h}$ after $70 \%$ PHx (Figure. 1). At 24, 72, 120, and $168 \mathrm{~h}$ after PHx, the liver regenerative ratios were $33.3 \pm$ 
$0.9 \%, 56.2 \pm 1.5 \%, 67.8 \pm 2.8 \%$ and $83.8 \pm 8.0 \%$, respectively.

\section{Portal and Systemic Plasma VEGF Levels}

Figure. 2 shows the systemic and portal venous plasma VEGF levels after 70\% PHx. Both the systemic and portal venous VEGF levels increased during $168 \mathrm{~h}$ after PHx. At 72, 120 and 168 $\mathrm{h}$ after PHx, the VEGF level was significantly higher in the portal venous blood than in the systemic venous blood (portal venous blood / systemic venous blood at 72, 120 and $168 \mathrm{~h}$ after PHx: $17.2 \pm 6.6 / 13.0 \pm$ 5.8, $20.2 \pm 9.7 / 12.8 \pm 6.6$ and $24.0 \pm 6.6 / 14.7 \pm 9.8$ pg/ml; $P=.003$, $P=.022$ and $P=.032$, respectively).

\section{VEGF mRNA Expression in the Liver, Spleen and Intestine}

Figure. 3 shows the ratios of VEGF mRNA expression compared to the corresponding pre-PHx levels in the liver (A), spleen (B) and intestine (C) during the $168 \mathrm{~h}$ after PHx. VEGF mRNA expression in the regenerating liver doubled at $72 \mathrm{~h}$ after PHx compared with the pre-PHx level. At 24, 72, 120 and $168 \mathrm{~h}$ after PHx, the ratios of VEGF mRNA expression were $1.48 \pm 0.07,2.03 \pm 0.19,1.97 \pm 0.24$ and $2.07 \pm 0.06$, respectively, and significantly higher than the pre-PHx level $(P=.016, P=.0051, P=.014$ and $P=.000027$, respectively).

VEGF mRNA expression in the spleen peaked at $72 \mathrm{~h}$ after PHx, and gradually decreased beyond $120 \mathrm{~h}$ after PHx (Figure. 3B). At 24, 72 and $120 \mathrm{~h}$ after PHx, the ratios of VEGF mRNA expression were $1.35 \pm 0.09,1.61 \pm 0.02$ and $1.26 \pm 0.02$, respectively, and significantly higher 
than the pre-PHx level $(P=.016, P=.000059$ and $P=.0045$, respectively).

VEGF mRNA expression in the intestine peaked at $72 \mathrm{~h}$ after PHx, and gradually decreased beyond $120 \mathrm{~h}$ after PHx (Figure. 3C). At 24 and $72 \mathrm{~h}$ after PHx, the ratios of VEGF mRNA expression were $1.19 \pm 0.03$ and $1.43 \pm 0.12$, respectively, and significantly higher than the pre-PHx level ( $P=.01$ and $P=.036$, respectively).

\section{HIF-1 $\alpha$ mRNA Expression in the Liver, Spleen and Intestine}

Figure. 4 shows the ratios of HIF-1 $\alpha$ mRNA expression compared to the corresponding pre-PHx levels in the liver (A), spleen (B) and intestine (C) during the 7 days after PHx.

HIF-1 $\alpha$ mRNA expression in the regenerating liver was more than 2-fold higher at $24 \mathrm{~h}$ after PHx compared to the pre-PHx level, and peaked at $72 \mathrm{~h}$ after PHx (Figure. 4A). At 24 and $72 \mathrm{~h}$ after PHx, the ratios of HIF- $1 \alpha$ mRNA expression were $2.26 \pm 0.10$ and $2.56 \pm 0.12$, respectively, and significantly higher than the pre-PHx level $(P=.019$ and $P=.012$, respectively).

HIF-1 $\alpha$ mRNA expression in the spleen peaked at $120 \mathrm{~h}$ after PHx (Figure. 4B). At 72 and 120 h after PHx, the ratios of HIF-1 $\alpha$ mRNA expression were $1.64 \pm 0.10$ and $1.62 \pm 0.12$, respectively, and significantly higher than the pre-PHx level $(P=.0019$ and $P=.047$, respectively).

HIF-1 $\alpha$ mRNA expression in the intestine peaked at $24 \mathrm{~h}$ after PHx, and decreased beyond 72 
h after PHx (Figure. 4C). At 24 and 72 h after PHx, the ratios of HIF-1 $\alpha$ mRNA expression were $1.31 \pm 0.07$ and $1.23 \pm 0.10$, respectively, and significantly higher than the pre-PHx level ( $P=.021$ and $P=.013$, respectively).

\section{HGF mRNA Expression in the Liver, Spleen and Intestine}

Figure. 5 shows the ratios of HGF mRNA expression compared to the corresponding pre-PHx levels in the liver (A), spleen (B) and intestine (C) after PHx.

HGF mRNA expression in the regenerating liver peaked at $120 \mathrm{~h}$ after PHx (Figure. 5A). At 24, 72 and $120 \mathrm{~h}$ after PHx, the ratios of HGF mRNA expression were $1.59 \pm 0.15,1.81 \pm 0.12$ and $2.04 \pm 0.14$, respectively, and significantly higher than the pre-PHx level $(P=.014, P$ $=.0036$ and $P=.0064$, respectively).

HGF mRNA expression in the spleen peaked at $120 \mathrm{~h}$ after PHx (Figure. 5B). At 24, 72 and $120 \mathrm{~h}$ after PHx, the ratios of HGF mRNA expression were $1.53 \pm 0.18,1.76 \pm 0.04$ and $1.94 \pm$ 0.07 , respectively, and significantly higher than the pre-PHx level $(P=.023, P=.043$ and $P$ $=.037$, respectively) .

HGF mRNA expression in the intestine gradually increased to the peak at $72 \mathrm{~h}$ after $\mathrm{PHx}$, and then gradually decreased (Figure. 5C). At $72 \mathrm{~h}$ after PHx, the ratio of HGF mRNA expression was $1.53 \pm 0.11$ and significantly higher than the pre-PHx level $(P=.027)$. 
Figure. 6 shows the ratios of HGFA mRNA expression compared to the corresponding pre-PHx levels in the liver (A), spleen (B) and intestine (C) during the 7 days after PHx.

HGFA mRNA expression in the regenerating liver gradually increased, and peaked at $72 \mathrm{~h}$ after PHx (Figure. 6A). At 72 and $168 \mathrm{~h}$ after PHx, the ratios of HGFA mRNA expression were $1.39 \pm 0.01$ and $1.14 \pm 0.11$, respectively, and significantly higher than the pre-PHx level $(P$ $=.0084$ and $P=.029$, respectively).

HGFA mRNA expression in the spleen peaked at $72 \mathrm{~h}$ after PHx (Figure. 6B). At 24, 72, 120 and $168 \mathrm{~h}$ after PHx, the ratios of HGFA mRNA expression were $1.05 \pm 0.02,1.26 \pm 0.08,1.30$ \pm 0.01 and $1.24 \pm 0.03$, respectively, and significantly higher than the pre-PHx level $(P=.0083$, $P=.023, P=.0026$ and $P=.013$, respectively).

HGFA mRNA expression in the intestine increased to 1.5-fold at $24 \mathrm{~h}$ after PHx compared to the pre-PHx level (Figure. 6C). At $24 \mathrm{~h}$ after PHx, the ratio of HGFA mRNA expression was $1.47 \pm 0.08$ and significantly higher than the pre-PHx level $(P=.045)$.

\section{DISCUSSION}

Previous studies have clarified that regenerating factors are essential for liver regeneration (11).

However, no studies have established that organs around the portal vein, such as the intestine and spleen, secrete liver regenerating factors into the portal venous blood during liver 
regeneration. We have reported that the portal venous blood VEGF level was higher than the systemic venous blood VEGF level during liver graft regeneration after LDLT and hypothesized that portal venous VEGF derived from the spleen or intestine plays an important role in liver graft regeneration, in addition to hepatocyte-derived VEGF (9).

Several authors have reported that VEGF secreted by proliferating hepatocytes represents an important stimulator of sinusoidal endothelial cell proliferation (12-14). Previous studies have reported that VEGF mRNA expression is markedly increased in the regenerating liver between 48 and 120 hours after hepatectomy, and gradually decreases thereafter $(14,15)$. Taniguchi et al. (13) demonstrated that VEGF mRNA expression is mainly detected in periportal hepatocytes by immunohistochemistry and in situ hybridization and suggested that VEGF promotes hepatocyte proliferation through the reconstruction of liver sinusoids via proliferation of sinusoidal endothelial cells. Shimizu et al. (15) also showed that VEGF secreted by replicating hepatocytes induces sinusoidal endothelial cell proliferation during regeneration after PHx in rats. Our results have revealed that not only the systemic VEGF level, but also the portal VEGF level, gradually increase during the first 168 hours after PHx. In addition, the portal venous VEGF levels were significantly higher than the systemic VEGF level for 72-168 h after PHx; consistent with our previous results for LDLT (9). These results suggest that portal venous VEGF plays an important role in liver regeneration after PHx. On the other hand, Bockhorn, M. 
(16) et al. has recently reported that VEGF is important during liver regeneration, however exogenous VEGF administration does not improve liver regeneration and survival liver regeneration after 90\% $\mathrm{PHx}$ in rats. For the next step, liver regeneration under VEGF administration from portal vein should be analyzed.

Hypoxia is well-known to be one of the strongest stimulants for angiogenesis, and HIF-1 $\alpha$ has been well recognized to play an essential role in hypoxic adaptation during mammalian development (17). Gopfert et al. (18) showed that moderate hypoxia enhances HIF-1 $\alpha$ expression in rat hepatocytes, while Maeno et al. (15) found that HIF-1 $\alpha$ expression is preceded by VEGF and fms-like tyrosine kinase-1 (flt-1) expressions and hypothesized that HIF-1 $\alpha$ is related to sinusoidal endothelial reconstruction. In the present study, VEGF mRNA expression was observed to occur after HIF-1 $\alpha$ mRNA expression in all the organs analyzed, and we therefore hypothesize that elevation of the portal venous pressure after PHx, which arises through shear stress, causes portal venous stasis that results in hypoxia in the viscera around the portal vein and consequently elevates the HIF-1 $\alpha$ level, thereby leading to VEGF production.

HGF is a heterodimeric protein that has been identified as a liver sinusoidal endothelial cell-derived paracrine mediator in liver regeneration (19). HGF is also produced by fibroblasts, hemocytes and endothelial cells in vitro and the spleen in vivo (20,21). In the present study, HGF mRNA expression was detected spleen and intestine as well as the regenerating liver after 
PHx. Although we were unable to analyze the systemic or portal venous HGF levels in the present study due to the limited blood samples available, our previous study revealed elevation of the HGF levels in the systemic and portal venous blood in LDLT recipients before and after transplantation (9). These findings may allow us to speculate that that portal venous HGF derived from the organs around the portal vein as well as HGF derived from sinusoidal endothelial cells induce liver regeneration.

On the other hand, Four proteases have been reported as factors that activate HGF in vitro, namely blood coagulation factor XIIa (22), urokinase (23), tissue-type plasminogen (24) and HGFA, which is most important for activating HGF (21). Miyazawa et al. (25) concluded that a proteolytic cascade plays a key role in the activation of HGF in response to tissue injury. After tissue injury, prothrombin is converted to thrombin, which activates HGFA, which in turn converts single-chain HGF to activated heterodimeric HGF (25). Kaido et al. (21) showed that HGFA protein is present in the liver, but not in the spleen or lungs after PHx in rats with liver cirrhosis. In the present study, however, HGFA mRNA was expressed not only in the regenerating liver, but also in intestine and spleen after PHx. Therefore, we could speculate that the HGFA up-regulated in the spleen and intestine activate the single-chain HGF in the portal venous blood, that cause hepatocytes to proliferate. Further studies are needed in order to more precisely define the role of portal venous regenerating factors secreted from the spleen or the 
intestine during liver regeneration after PHx or LDLT.

In conclusion, portal venous VEGF protein level was significantly higher than systemic venous VEGF during liver regeneration. In addition, expression of VEGF, HIF-1 $\alpha$, HGF, and HGFA mRNA in spleen and intestine were up-regulated during liver regeneration. These results suggest that hepatic regenerating factors derived from the spleen or intestine may contribute liver regeneration. 


\section{REFERENCES}

1. Nakamura, T., Nishizawa, T., Hagiya, M., et al. Molecular cloning and expression of human hepatocyte growth factor. Nature 1989;342:440-443.

2. Kan, M., Huang, J. S., Mansson, P. E., et al. Heparin-binding growth factor type 1 (acidic fibroblast growth factor): a potential biphasic autocrine and paracrine regulator of hepatocyte regeneration. Proc Natl Acad Sci U S A 1989;86:7432-7436.

3. McGowan, J. A., Strain, A. J., and Bucher, N. L. DNA synthesis in primary cultures of adult rat hepatocytes in a defined medium: effects of epidermal growth factor, insulin, glucagon, and cyclic-AMP. J Cell Physiol 1981;108:353-363.

4. Miyazawa, K., Shimomura, T., Kitamura, A., et al. Molecular cloning and sequence analysis of the cDNA for a human serine protease reponsible for activation of hepatocyte growth factor. Structural similarity of the protease precursor to blood coagulation factor XII. J Biol Chem 1993;268:10024-10028.

5. Mochida, S., Ishikawa, K., Inao, M., et al. Increased expressions of vascular endothelial growth factor and its receptors, flt-1 and KDR/flk-1, in regenerating rat liver. Biochem Biophys Res Commun 1996;226:176-179.

6. Slapak, M., Wexler, M., Mizumoto, R., et al. The role of arterial portal and systemic venous blood on regeneration of the canine liver. Br J Surg 1970;57:392. 
7. Wexler, M., Slapak, M., Mizumoto, R., et al. Regeneration and maintenance of integrity of canine liver. The role of arterial, portal, and systemic venous blood. Arch Surg 1970;101:267-276.

8. Starzl, T. E., Porter, K. A., and Kashiwagi, N. Portal hepatotrophic factors, diabetes mellitus and acute liver atrophy, hypertrophy and regeneration. Surg Gynecol Obstet 1975;141:843-858.

9. Yagi, S., Iida, T., Taniguchi, K., et al. Impact of portal venous pressure on regeneration and graft damage after living-donor liver transplantation. Liver Transpl 2005;11:68-75.

10. Hinngis G, A. R. Restoration of the liver the white rat following partial surgical removal. Arch Pathol 1931;12:186-202.

11. Bucher, N. L. Regeneration of Mammalian Liver. Int Rev Cytol 1963;15:245-300. 12. Assy, N., Spira, G., Paizi, M., et al. Effect of vascular endothelial growth factor on hepatic regenerative activity following partial hepatectomy in rats. J Hepatol 1999;30:911-915. 13. Taniguchi, E., Sakisaka, S., Matsuo, K., et al. Expression and role of vascular endothelial growth factor in liver regeneration after partial hepatectomy in rats. J Histochem Cytochem 2001;49:121-130.

14. Shimizu, H., Miyazaki, M., Wakabayashi, Y., et al. Vascular endothelial growth 
factor secreted by replicating hepatocytes induces sinusoidal endothelial cell proliferation

during regeneration after partial hepatectomy in rats. J Hepatol 2001;34:683-689.

15. Maeno, H., Ono, T., Dhar, D. K., et al. Expression of hypoxia inducible factor-1alpha during liver regeneration induced by partial hepatectomy in rats. Liver Int 2005;25:1002-1009.

16. Bockhorn, M., Schollmann, S., Opitz, B., et al. Vascular endothelial growth factor does not improve liver regeneration and survival after 90\% subtotal liver resection. Hepatol Res 2007;37:353-359.

17. Iyer, N. V., Kotch, L. E., Agani, F., et al. Cellular and developmental control of O2 homeostasis by hypoxia-inducible factor 1 alpha. Genes Dev 1998;12:149-162.

18. Gopfert, T., Gess, B., Eckardt, K. U., and Kurtz, A. Hypoxia signalling in the control of erythropoietin gene expression in rat hepatocytes. J Cell Physiol 1996;168:354-361.

19. LeCouter, J., Moritz, D. R., Li, B., et al. Angiogenesis-independent endothelial protection of liver: role of VEGFR-1. Science 2003;299:890-893.

20. Matsumoto, K., Tajima, H., Okazaki, H., and Nakamura, T. Negative regulation of hepatocyte growth factor gene expression in human lung fibroblasts and leukemic cells by transforming growth factor-beta 1 and glucocorticoids. J Biol Chem 1992;267:24917-24920. 21. Kaido, T., Oe, H., Yoshikawa, A., et al. Expressions of molecules associated with hepatocyte growth factor activation after hepatectomy in liver cirrhosis. Hepatogastroenterology 
2004;51:547-551.

22. Shimomura, T., Miyazawa, K., Komiyama, Y., et al. Activation of hepatocyte growth factor by two homologous proteases, blood-coagulation factor XIIa and hepatocyte growth factor activator. Eur J Biochem 1995;229:257-261.

23. Naldini, L., Tamagnone, L., Vigna, E., et al. Extracellular proteolytic cleavage by urokinase is required for activation of hepatocyte growth factor/scatter factor. Embo J 1992;11:4825-4833.

24. Mars, W. M., Zarnegar, R., and Michalopoulos, G. K. Activation of hepatocyte growth factor by the plasminogen activators uPA and tPA. Am J Pathol 1993;143:949-958. 25. Miyazawa, K., Shimomura, T., and Kitamura, N. Activation of hepatocyte growth factor in the injured tissues is mediated by hepatocyte growth factor activator. J Biol Chem 1996;271:3615-3618. 